\title{
Informationen für die Klinik
}

\section{Notfallmedizin}

Mund-zu-Mund-Beatmung mit Infektionsschutz

Speziell die Mund-zu-Nase- und Mund-zu-Mund-Beatmung birgt für den Notfall-Hel-fer das Risiko, sich mit gefährlichen Infek-tionserregern, wie z. B. AIDS- oder Hepati-tis-B-Viren, zu infizieren. Diese Problematik hat die Firma Weinmann zum Anlaß genommen, den «Lifeway» mit Infektions-Schutz zu entwickeln. Erzielt wird dieser Schutz-Effekt durch ein inte-griertes Sicherheitsventil, ein Nichtrück-atemventil. Es sitzt zwischen dem Einblas-Mundstück und dem Tubus. Bei der Beat-mung durch den Heifer gelangt der Luft-strom durch das EinblasMundstück, die sich öffnende Lippenmembran und den Tubus bis in das Bronchialsystem und in die Lungen des Patienten. Bei der Exspiration des Patienten verläuft der Luftstrom genau entgegengesetzt: zurück durch den Tubus bis an die Lippenmembran. Dort sperrt die Lippenmembran die Luft in Richtung Einblas-Mundstück und leitet sie über Luftaus-trittsschlitze nach unten ins Freie ab. Die Handhabung ist einfach. Der integrierte Spezialtubus halt die Zunge des Verunglück-ten zurück. Eine Markierung auf der Mund-abschlußkappe erleichtert das richtige Ein-führen. Die Nasenklemme ist mit einer Ver-lustsicherung an den «Lifeway» befestigt. Weitere Informationen bei Firma Weinmann, Kronsaalsweg 40, D-2000 Hamburg 54, Tel.: 040/540092-0.

«Lifeway»-Beatmungshilfe mit Infektionsschutz

Bücher

Gottfried Benn zum 100. Geburtstag

Die Krewel-Werke bieten aus Anlaß des hundertsten Geburtstages des Lyrikers und Essayisten Gottfried Benn interessierten Ärzten das Taschenbuch «Begegnungen mit Gottfried Benn» an. Dem Verfasser gelang es, eine Brücke zu schlagen zu namhaften Persönlichkeiten aus Kultur und Wissen-schaft.

Das Benn-Buch gibt es kostenlos bei den Krewel-Werken GmbH, Krewelstraße 2, D-5208 Eitorf.

Infusionstherapie

Neue Infusionsregler I

Der neue Rateminder ${ }^{\circledR}$ wird einfach an die Tropfenkammer eines normalen StandardInfusionsbestecks gehängt. Aufwendige Schlauchsysteme entfallen. Dank Akku-Be-trieb arbeitet Rateminder ${ }^{\circledR}$ über $25 \mathrm{~h}$ unab-hängig vom Netzstrom. Die differenzierte Alarmfunktion und ein redundantes, elektronisch gesteuertes Sicherheitssystem mit automatischer Ab-schaltung bei Fehlfunktion verringern das Risiko der extravasalen Infiltration. Der Rateminder ${ }^{\circledR}$ ermöglicht eine kontrol-lierte und kontinuierliche Niederdruck-Infu-sionstherapie durch Vorwahl der definierten Infusionsrate von 1-99 Tropfen/min (1-300 ml/h) und präzise Regelung der Schwerkraft-Infusion.

Die Tropfenzahlgenauigkeit beträgt 99\%, während Standard-Schwerkraft-Infusionen Abweichungen von bis zu 50\% zeigen. Weitere Informationen erhalten Sie durch: Critikon GmbH, Fachbereich Infusionstech-nik, Mühlenweg 143, D-2000 Norderstedt, Tel. 040/5260010. 
Neue Infusionsregler II

IVAC stellt den neuen volumetrischen Schwerkraft-Infusionsregler Modell 280 vor, der speziell für den Einsatz auf Allgemein-stationen ausgelegt ist. Der Förderbereich liegt bei 1-299 ml/h, ein-stellbar in 1-ml-Schritten. Bei Abweichungen der Förderrate wird die Infusion auto-matisch unterbrochen und ein optischer wid akustischer Alarm ausgelöst. Die erf order-lichen

Bedienungsschritte wie auch auftre-tende Störungen werden direkt auf einem Display angezeigt. Die verschiedenen Alarmfunktionen werden optisch und aku-stisch ausgelöst. Die integrierte Batterie ist Standard und ermöglicht netzunabhängigen

Betrieb bis zu 6 h. Ein Voralarm erfolgt ca. lh vor Entladung der Batterie. Ferner ist die Möglichkeit der Zählung der Betriebs-stunden gegeben, was speziell im Hinblick auf die Forderungen der MedGV einen opti-malen Wartungsrhythmus gewährleistet. IVAC Medizintechnik GmbH, Teichweg 3, 6300 Giessen

Produktinformation

Neue Fettemulsion von Abbott

Die Firma Abbott bietet seit Juni 1986 erst-mals eine Fettemulsion auch in Deutschland an, welche aufgrund ihrer hohen klinischen Akzeptanz in den USA sehr weit verbreitet ist. Sie enthält als Wirkstoffkomponente eine neuartige Kombination aus 50\% Soja-bohnen- und 50\% Safloröl. Mit dieser Mischung wird ein sehr hoher Gehalt $(65,8 \%)$ an Linolsäure erreicht, der für den menschlichen Körper wichtigsten essentiellen Fettsäure. Der ebenfalls aus dieser Kombination resultierende niedrige Linolensäure-Gehalt von 4,2\% führt insge-samt zu einem günstigen Linolen-/Linolsäu-re-Verhältnis von 1:16. Dies ist von Bedeu-tung, da Linolensäure das linolsäureverar-beitende Enzymsystem hemmt. Da Linolsäure die Ausgangssubstanz zur Biosynthese der für den Organismus wichtigen Prosta-glandine, Prostazykline und Thromboxane der 2er-Serie (z.B. A2, E2 und F2) darstellt, kommt der 65,8\%ige Linolsäuregehalt der neuen Fettemulsion dem besonders nach Operationen oder bei Trauma erhöhten Bedarf des Körpers entgegen. Der hohe Gehalt an Vitamin E ( $\alpha$-Toco-pherol) schützt als natürliches Antioxidans die essentiellen Fettsäuren und die Zell-membranen vor oxidativen Veränderungen. Dies ist für die gute Verträglichkeit ebenso wichtig wie der sehr niedrige Cholesteringe-halt dieser neuen Emulsion. Klinisch relevant ist auch, daß Heparin der Emulsions-Flasche direkt zugegeben werden kann. Heparin erhöht bekanntlich die Fettclearancerate durch Enzymaktivierung. Detaillierte Informationen sind über die fol-gende Adresse erhältl-ich: Deutsche Abbott GmbH, Max-Planck-Ring 2, Delkenheim, D-6200 Wiesbaden

Preisausschreiben

Konrad-Lang-Preis

Die Deutsche Arbeitsgemeinschaft für künstliche Ernährung (DAKE) und die 